\title{
Application of Nursing Risk Management in Nursing of Severe Peptic Ulcer Bleeding
}

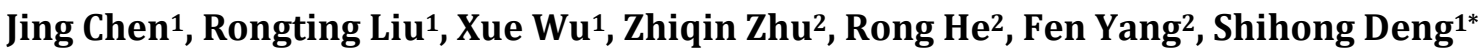 \\ ${ }^{1}$ Department of Gastrointestinal Surgery, The First Affiliated Hospital of Yangtze University, Jingzhou, China \\ ${ }^{2}$ Department of Gastroenterology, The First Affiliated Hospital of Yangtze University, Jingzhou, China \\ Email: ^382209608@qq.com
}

How to cite this paper: Chen, J., Liu, R.T., Wu, X., Zhu, Z.Q., He, R., Yang, F. and Deng, S.H. (2021) Application of Nursing Risk Management in Nursing of Severe Peptic Ulcer Bleeding. Yangtze Medicine, 5, 226-234.

https://doi.org/10.4236/ym.2021.53021

Received: July 16, 2021

Accepted: September 14, 2021

Published: September 17, 2021

Copyright $\odot 2021$ by author(s) and Scientific Research Publishing Inc. This work is licensed under the Creative Commons Attribution International License (CC BY 4.0).

http://creativecommons.org/licenses/by/4.0/

\begin{abstract}
Objective: To assess and compare the clinical application value of nursing risk management in the diagnosis and treatment of severe peptic ulcer bleeding. Methods: A total of 70 patients with severe peptic ulcer bleeding were selected as the research objects, randomly divided into observation group and control group, 35 cases for each. The nursing risk management and conventional nursing management were implemented for the two groups, respectively. The success rate of hemostasis, average length of stay, cure rate, complication rate, dyspnea rate, nursing dispute rate, SAS score, and SDS score were compared between the two groups. Patients' satisfaction with nursing quality was also compared. Results: After the implementation of two different nursing management methods, all the above-mentioned metrics were better in the observation group than in the control group, and the differences were statistically significant $(\mathrm{P}<0.01$ or $\mathrm{P}<0.05)$. Conclusion: The application of nursing risk management in the nursing intervention process of patients with severe peptic ulcer bleeding can effectively reduce the probability of nursing risk events, increase the cure rate, shorten the patient's hospital stay, but also improve the patients' psychological state and increase the patient's satisfaction with nursing quality, which is worth clinical promotion.
\end{abstract}

\section{Keywords}

Risk Management, Peptic Ulcer, Upper Gastrointestinal Bleeding, Nursing

\section{Introduction}

Peptic ulcer is one of the main causes of non-varices gastrointestinal bleeding [1] [2]. It is also a ubiquitous type of disease in clinical gastroenterology, and often complicates with gastrointestinal bleeding. Patients with severe peptic ulcer can bleed more than $1000 \mathrm{~mL}$ within a few hours, or the blood loss reaches $1 / 5$ of the 
total circulating blood volume. It may put life threatening on patients in the case that the bleeding cannot be stopped in time and effective intervention measures are not taken [3] [4]. As peptic ulcer bleeding (PUB) is complicated and variable, with many uncertain factors, it has a higher risk. Patients with severe peptic ulcer bleeding, in particular, are prone to depression, anxiety and other unhealthy emotions due to the long course and recurrence of the disease, which seriously affects the clinical diagnosis and treatment effect and prognosis [5]. Nursing risk management is a management system through which clinical nurses can effectively identify potential nursing risk events, analyze and select them, intervene in advance to deal with direct or indirect death or disability risk factors that may threaten safety, and establish and implement targeted nursing management measures to reduce the probability of risk events and avoid risk events that will do harm to doctors and patients [6] [7]. In this study, we intend to implement nursing risk management for patients with severe peptic ulcer bleeding to assess its clinical nursing application value and its influence on patients' negative psychological state.

\section{Method Part}

\subsection{Case Information}

A total of 70 patients, who were admitted to our hospital between January 2019 and January 2020 with severe peptic ulcer bleeding, were selected as the research objects. They were divided into observation group and control group using random number table and random number reminder methods, 35 cases for each group.

The two groups were implemented nursing risk management and conventional nursing management, respectively. There were 24 males and 11 females in the observation group, with an average age of $53.62 \pm 5.23$ years old. The control group had 26 males and 9 females, aged $52.67 \pm 4.85$ years old in average. There was no significant difference in age and sex between the two groups. Inclusion criteria: 1) all the selected patients had clear diagnoses after relevant auxiliary examinations, with over $1000 \mathrm{~mL}$ blood loss within 24 hours; 2) they were well communicated with clear consciousness; 3) the clinical and nursing data are complete without any loss; 4) patients participated in the whole process. Exclusion criteria: 1) patients with HIV infection and active tuberculosis that endanger the safety of researchers; 2 ) those who were unable to cooperate with nursing workers on their own; 3) those who died or withdrew midway. This study obtained informed consent from all participating patients and their families, and reported to the Ethics Committee of Jingzhou First People's Hospital for review and approval.

\subsection{Methods}

\subsubsection{Control Group: Conventional Nursing Management Intervention}

Patients in the control group, after admission, were implemented conventional 
nursing management according to the clinical nursing requirements of severe peptic ulcer bleeding. The main measures included close monitoring of the patient's vital signs, blood loss nursing based on the patient's condition, timely drug delivery following the doctor's advice, replenishing blood volume, closely observing the properties of vomit and excrement, keeping the airway unobstructed, etc.

\subsubsection{Observation Group: Risk Management Program}

The observation group was implemented risk management. The content of the risk management program contained the assessment of disease risk events and the assessment of emotional state risk events. Both were included in the overall risk assessement indexes, and then nursing risk management measures were implemented according to the assessment results.

\section{1) Assessment of Disease Risk Events}

The risk events that may occur during diagnosis and treatment process of patients in the observation group were screened and formulated based on their conditions after admission to form the overall risk assessment indexes [8] [9]. A total score $<9$ was considered low risk, 9 - 12 considered medium risk, and $>12$ considered high risk. Forrest grading [8], Glasgow-Blatchford score (GBS) [10], and Rockall score [11] were combined to form the risk assessment of disease severity (RODS), which was included in the overall risk assessment (Table 1).

\section{2) Assessment of Emotional State Risk Events}

According to the patient's score obtained from the self-rating depression scale (SDS) [12] and the self-rating anxiety scale (SAS) [13], the emotional state assessment scale (MSAS) was formed. SDS and SAS scores are based on a 4-level scoring system [14]. The SDS total score $<53$ was considered normal, $53-62$ considered mild depression, 63 - 72 considered medium depression, and $>72$

Table 1. Overall risk assessment indexes.

\begin{tabular}{cccc}
\hline \multirow{2}{*}{ Assessment indexes } & \multicolumn{3}{c}{ Score } \\
\cline { 2 - 4 } Age & 1 & 2 & 3 \\
\hline Complication & None & 1 complication & 2 or above complications \\
Maximum diameter of ulcer & $\leq 1 \mathrm{~cm}$ & $1-2 \mathrm{~cm}$ & $>2 \mathrm{~cm}$ \\
Shock index & $<0.7$ & $0.7-0.9$ & $>0.9$ \\
Pain degree & Mild & Moderate & Severe \\
RODS & Forrest III & Forrest IIa - IIc & Forrest Ia - Ib \\
& GBS $\leq 6$ & GBS $7-9$ & GBS $\geq 10$ \\
MSAS & Rockall $\leq 2$ & Rockall $3-4$ & Rockall $\geq 10$ \\
& SDS $<53$ & SDS $53-62$ & SDS $\geq 63$ \\
& SAS $<50$ & SAS $50-60$ & SAS $\geq 61$ \\
\hline
\end{tabular}

Note: Pain degree used visual analogue scale. Score 1 - 3 was considered mild, 4 - 6 considered moderate, and 7 - 10 considered severe. 
considered severe depression. As to the SAS score, the total score $<50$ was considered normal, 50 - 59 considered mild anxiety, 60 - 69 considered medium anxiety, and $>69$ considered severe anxiety. As a result, in MSAS, the low risk was SDS $<53$ and/or SAS $<50$, the medium risk was SDS $\leq 62$ and/or SAS $\leq 59$, and the severe risk was SDS $>62$ and/or SAS $>60$, which was also included in the overall risk assessment (Table 1).

3) Implementation of Nursing Risk Management Measures Based on the Assessment Results

Based on the assessment results, we combined the main points of the daily work of specialized nursing to analyze and assess possible risk events, and clarify the risk management indexes and priority levels in accordance with harmful level. Then we formulated nursing risk management measures according to the risk assessment level and implement them in different levels. The nurses of the observation group strengthened professional skills training, and conduct regular assessments to enhance their sense of responsibility and professional skills. 1) Low-risk nursing: on the basis of conventional nursing, the nurses focus on strengthening psychological nursing and health education. In view of the change or aggravation of the patient's condition that brings psychological pressure and tension to the patient, and even psychological problems such as depression and anxiety, the responsible nurse should make early warning and intervention in reference to the assessment points, maintain close communication with patients and their families, help patients to establish confidence and hope to overcome the disease and to stay optimistic, and eliminate the influence of unhealthy emotions on patients. In addition, the responsible nurse should emphasize the necessity of hemostatic drugs, somatostatin and other drugs, and do a good job in diet care and health education. 2) Medium-risk nursing: the focus of nursing was to closely monitor risk factors of bleeding, especially pay attention to the observation of bleeding peak time nodes (5:00 am-7:00 am, 5:00 pm-12:00 pm), carefully identify signs of rebleeding, strengthen ward inspections, maintain communication between doctors and patients, and monitor changes in vital signs. For emotionally unstable patients, special personnel should be arranged to accompany them, and psychological support should be provided. Their emotional states should be reported to doctors if necessary, so as to apply for intervention by a psychologist. 3) Severe-risk nursing: the focus of nursing was to prepare for emergency nursing in advance to deal with possible bleeding and hemorrhage at any time, including emergency equipment and hemostatics, closely monitor vital signs following the doctor's instructions, carefully perform ECG monitoring, rapidly establish venous channels, get ready for blood transfusion, and count the success rate of hemostasis of the two groups. Nurses should take good nursing measures, and instruct the patient to stay in bed absolutely and turn his head to one side to avoid inhalation suffocation. During the hemostasis, nurses still need to remain vigilant to avoid rebleeding of patients. An inspection every 15 - 30 min was required and the nursing content should be recorded. Additionally, it was as well to closely monitor the patient's unhealthy emotions. Nurses must 
report to the doctor immediately once abnormalities were found, and give sedative intervention as appropriate following the doctor's advice.

\subsubsection{Observation Indexes}

When patients were discharged, the differences in nursing risk events between the two groups were collected and compared, including the success rate of hemostasis, average hospital stay, cure rate, complication rate, dyspnea rate, nursing dispute rate, and SAS and SDS scores. Meanwhile, the nursing satisfaction questionnaires were designed to conduct survey, containing emergency nursing ability, disease observation ability, hemostasis and rescue item intact rate, accident rate, bleeding treatment ability, nursing promotion and education, etc. The point system was used. The total score was 100 points, $81-100$ represented excellent, 60 - 80 represented qualified, and less than 60 represented unqualified. Nursing satisfaction $=$ (excellent + qualified $)$ number of cases/total number of cases $\times 100 \%$. Through the above indexes, the impact of nursing risk management measures on patients with severe peptic ulcer bleeding was analyzed and compared.

\subsubsection{Statistical Analyses}

SPSS20.0 Software was utilized for data analysis. Measurement data were expressed as mean $\pm \mathrm{SD}$, and t test was used for comparison between groups. Count data is expressed by percentage (\%), and $\chi^{2}$ test was used for comparison between groups. A value of $\mathrm{P}<0.05$ was considered statistically significant, and $\mathrm{P}<0.01$ was considered very significant.

\section{Results}

\subsection{Comparison of Differences in Nursing Risk Events}

Compared with the control group, the success rate of hemostasis and cure rate in the observation group were obviously increased $(\mathrm{P}<0.05)$, and the difference was significant $(\mathrm{P}<0.05)$. Such indexes as the complication rate, dyspnea, and nursing dispute rate were significantly reduced $(\mathrm{P}<0.05)$ (Table 2$)$. The length of stay was shorter in contrast to the control group, and the SAS score and SDS score were lower than those of the control group. The differences were significant $(\mathrm{P}<0.05)$ (Table 3).

Table 2. Comparison of the probability of nursing risk events between the observation group and the control group.

\begin{tabular}{ccccc}
\hline Risk factors & $\begin{array}{c}\text { Observation group } \\
\mathrm{n}(\%)\end{array}$ & $\begin{array}{c}\text { Control group } \\
\mathrm{n}(\%)\end{array}$ & $\chi^{2}$ value & $\mathrm{P}$ value \\
\hline Success rate of hemostasis & $33(94.29)$ & $30(85.71)$ & 7.12 & $\mathrm{P}<0.05$ \\
Cure rate & $28(80.00)$ & $24(68.57)$ & 7.23 & $\mathrm{P}<0.05$ \\
Complication rate & $2(5.71)$ & $5(14.29)$ & 6.22 & $\mathrm{P}<0.01$ \\
Dyspnea rate & $1(2.86)$ & $3(8.57)$ & 9.86 & $\mathrm{P}<0.05$ \\
Nursing dispute rate & $0(0)$ & $5(14.29)$ & 6.74 & $\mathrm{P}<0.01$ \\
\hline
\end{tabular}


Table 3. Comparison of average length of stay and psychological state score between the observation group and the control group.

\begin{tabular}{ccccc}
\hline Group & Number of cases & Average length of stay & SAS score & SDS score \\
\hline The observation group & 35 & $9.89 \pm 2.68^{*}$ & $41.65 \pm 5.31^{*}$ & $36.68 \pm 4.26^{*}$ \\
The control group & 35 & $15.65 \pm 3.25$ & $48.88 \pm 5.56$ & $43.65 \pm 5.12$ \\
\hline
\end{tabular}

Note: The differences between the observation group and the control group were statistically significant ${ }^{*} \mathrm{P}$ $<0.05)$.

\subsection{Comparison of Nursing Quality Satisfaction}

The nursing quality satisfaction of the observation group was much higher than that of the control group, and the difference was significant $(\mathrm{P}<0.05)$ (Table 4).

\section{Discussion}

Severe peptic ulcer bleeding is a ubiquitous clinical gastroenterology disease, with a high morbidity. Patients are often in unstable conditions, and their digestive function is largely affected by the pathological lesion of the digestive system [15]. Endoscopy technology has made rapid progress, new proton pump inhibitors have also begun to be widely used in clinics, and the proportion of severe peptic ulcer bleeding cases requiring surgical treatment has been declining year by year, but the bleeding problem still plagues clinicians, and the nursing of patients with severe peptic ulcer bleeding, consequently, also poses a serious challenge. Nursing risk management is a standardized predictive management procedure, which can well prevent risk events in the conventional management process [6] [7]. However, there is still a lack of reference on how to establish effective detailed rules for nursing assessment. In our research, we, for the first time, worked closely with clinicians, and referred to the relevant risk factors related to early rebleeding in patients with severe peptic ulcer bleeding as well as the relevant requirements of the clinician's "Guidelines for the diagnosis and treatment of acute non-varices upper gastrointestinal bleeding" [8] [9] to form the detailed rules for overall risk assessment, and try to assess the risk events in the nursing management process according to the rules, and grade the nursing management according to the risk degree, which provides a practical assessment basis for standardizing specialized nursing management.

The implementation of the detailed rules requires that high-quality nurses carry out on ground. In this study, the rules were implemented at different levels according to the risk degree during the nursing process of patients with severe peptic ulcer bleeding. The results showed that in contrast to the control group, the success rate of hemostasis and the cure rate were significantly higher in the observation group, the complication rate, dyspnea and nursing dispute rate were significantly reduced, and the length of stay was also obviously shortened. The results indicate that the overall risk assessment indexes have certain clinical practical reference value; on the other hand, early predictive nursing risk management can effectively improve the hemostasis effect and reduce the risk of 
Table 4. Comparison of the satisfaction with nursing quality between the observation group and the control group.

\begin{tabular}{ccccc}
\hline Group & $\begin{array}{c}\text { Number } \\
\text { of cases }\end{array}$ & $\begin{array}{c}\text { Number of } \\
\text { result excellent }\end{array}$ & $\begin{array}{c}\text { Number of } \\
\text { result qualified }\end{array}$ & $\begin{array}{c}\text { Satisfaction } \\
\text { rate (\%) }\end{array}$ \\
\hline The observation group & 35 & 22 & 11 & 94.29 \\
The control group & 35 & 15 & 14 & 82.86 \\
\hline
\end{tabular}

Note: The difference between the observation group and the control group was statistically significant $\left(\chi^{2}=\right.$ 6.71, $\mathrm{P}<0.05)$

blood loss. In addition, the implementation of nursing risk management measures can also effectively affect the probability of nursing risk events, increase the cure rate, and shorten the patient's hospital stay.

Patients with severe peptic ulcer bleeding are prone to depression anxiety and other unhealthy emotions due to the long course of disease, the recurrence of the disease, and the tendency to bleed. In view of this risk event, corresponding nursing measures were implemented. The results showed that the psychological state score of the observation group was better than that of the control group, indicating that the nursing risk management measures can alleviate patients' unhealthy emotions, with a better nursing effect.

The main purpose of the introduction of the nursing risk management system is to reduce nursing risks and improve nursing quality fundamentally. In our research, we implement nursing risk management measures through professional skill training, regular examination, satisfaction survey questionnaires, and the like. The results showed that the observation group's satisfaction with nursing quality significantly increased, suggesting that these measures can effectively enhance the communication between doctors and nurses, nurses and patients, and improve nurses' professional skills, thereby effectively reducing the probability of nursing risk, eliminating the hidden dangers of nursing safety, and improving the resilience of nurses.

\section{Conclusion}

In a nut shell, the application of nursing risk management in the nursing intervention process of patients with severe peptic ulcer bleeding can effectively reduce the probability of nursing risk events, increase cure rate, shorten patient's hospital stay, but also improve the patient's psychological state and satisfaction with nursing, which is worthy of promotion in clinical practice.

\section{Acknowledgements}

This work was supported by grants from the Jingzhou Science and Technology Development Plan (2020HC28).

\section{Conflicts of Interest}

The authors declare no conflicts of interest regarding the publication of this paper. 


\section{References}

[1] Mujtaba, S., Chawla, S. and Massaad, J.F. (2020) Diagnosis and Management of Non-Variceal Gastrointestinal Hemorrhage: A Review of Current Guidelines and Future Perspectives. Journal of Clinical Medicine, 9, 402. https://doi.org/10.3390/jcm9020402

[2] De Angelis, C.G., Cortegoso Valdivia, P., Rizza, S., Venezia, L., Rizzi, F., Gesualdo, M., Saracco, G.M. and Pellicano, R. (2020) Endoscopic Ultrasound-Guided Treatments for Non-Variceal Upper GI Bleeding: A Review of the Literature. Journal of Clinical Medicine, 9, 866. https://doi.org/10.3390/jcm9030866

[3] Nelms, D.W. and Pelaez, C.A. (2018) The Acute Upper Gastrointestinal Bleed. The Surgical Clinics of North America, 98, 1047-1057. https://doi.org/10.1016/j.suc.2018.05.004

[4] Schmidt, A.R., Glaser, N., Kuellmer, A., Caca, K. and Lau, J. (2020) The Use of the over the Scope Clip to Treat Upper Gastrointestinal Bleeding. Gastrointestinal Endoscopy Clinics of North America, 30, 1-11. https://doi.org/10.1016/i.giec.2019.08.001

[5] Farrar, F.C. (2018) Management of Acute Gastrointestinal Bleed. Critical Care Nursing Clinics of North America, 30, 55-66. https://doi.org/10.1016/j.cnc.2017.10.005

[6] Slemon, A., Jenkins, E. and Bungay, V. (2017) Safety in Psychiatric Inpatient Care: The Impact of Risk Management Culture on Mental Health Nursing Practice. Nursing Inquiry, 24, e12199. https://doi.org/10.1111/nin.12199

[7] Coronado-Vázquez, V., García-López, A., López-Sauras, S. and Turón Alcaine, J.M. (2017) Nursing Involvement in Risk and Patient Safety Management in Primary Care. Implicación de las enfermeras en la gestión de riesgos y la seguridad del paciente en Atención Primaria. Enfermeria Clinica, 27, 246-250.

https://doi.org/10.1016/j.enfcle.2017.04.006

[8] de Groot, N.L., van Oijen, M.G., Kessels, K., Hemmink, M., Weusten, B.L., Timmer, R., Hazen, W.L., van Lelyveld, N., Vermeijden, R.R., Curvers, W.L., Baak, B.C., Verburg, R., Bosman, J.H., de Wijkerslooth, L.R., de Rooij, J., Venneman, N.G., Pennings, M., van Hee, K., Scheffer, B.C., van Eijk, R.L., Bredenoord, A.J., et al. (2014) Reassessment of the Predictive Value of the Forrest Classification for Peptic Ulcer Rebleeding and Mortality: Can Classification Be Simplified?. Endoscopy, 46, 46-52. https://doi.org/10.1055/s-0033-1344884

[9] Bai, Y. and Li, Z.S. (2019) Standardize the Diagnosis and Treatment of Acute Non-Variceal Upper Gastrointestinal Bleeding Based on the Update Guidelines. Chinese Journal of Internal Medicine, 58, 161-163.

[10] Uysal, Y., Babus, S.B., Kose, A., Ates, F., Biricik, S., Erdogan, S., Cevik, I., Toker, I. and Ayrik, C. (2019) The Prognostic Significance of the Risk Scores at Upper Gastrointestinal Bleeding. Nigerian Journal of Clinical Practice, 22, 1099-1108.

[11] Kim, M.S., Choi, J. and Shin, W.C. (2019) AIMS65 Scoring System Is Comparable to Glasgow-Blatchford Score or Rockall Score for Prediction of Clinical Outcomes for Non-Variceal Upper Gastrointestinal Bleeding. BMC Gastroenterology, 19, 136. https://doi.org/10.1186/s12876-019-1051-8

[12] Jokelainen, J., Timonen, M., Keinänen-Kiukaanniemi, S., Härkönen, P., Jurvelin, H. and Suija, K. (2019) Validation of the Zung Self-Rating Depression Scale (SDS) in Older Adults. Scandinavian Journal of Primary Health Care, 37, 353-357. https://doi.org/10.1080/02813432.2019.1639923

[13] Morard, M.D., Gonzalez-Monge, S., Rippert, P., Roche, S., Bernard, J.C., Lagauche, 
D., Delvert, C., Luauté, J., Jacquin-Courtois, S., Caillet, F., Di Marco, J., Ghelfi, F., Otmani, S., Calmels, P., Royet, M., Joseph, P.A., Ecochard, R., Rode, G., Vuillerot, C. and SAS Study Group (2018) Construction and Feasibility Study of the SOFMER Activity Score (SAS), a New Assessment of Physical and Cognitive Activity. Annals of Physical and Rehabilitation Medicine, 61, 315-322.

https://doi.org/10.1016/j.rehab.2018.04.006

[14] Yue, T., Li, Q., Wang, R., Liu, Z., Guo, M., Bai, F., Zhang, Z., Wang, W., Cheng, Y. and Wang, H. (2020) Comparison of Hospital Anxiety and Depression Scale (HADS) and Zung Self-Rating Anxiety/Depression Scale (SAS/SDS) in Evaluating Anxiety and Depression in Patients with Psoriatic Arthritis. Dermatology (Basel, Switzerland), 236, 170-178. https://doi.org/10.1159/000498848

[15] Busch, M., Schneider, A., Lankisch, T. and von Hahn, T. (2017) Akute Blutung im oberen Gastrointestinaltrakt [Acute Hemorrhage in the Upper Gastrointestinal Tract]. Der Internist, 58, 226-232. https://doi.org/10.1007/s00108-016-0184-X 